\title{
Pensar lo múltiple sin el concepto: hacia un intelecto democrático*
}

\author{
David Webh** \\ Traducción del francés al español de Luis Alfonso Paláu-Castaño \\ Universidad Nacional de Colombia, Medellín, Colombia \\ lapalau@gmail.com
}

Desde las primeras páginas de Génesis, Michel Serres (1982) expone su objeto: hablar de lo múltiple, incluso sin recurrir al concepto (p. 4). Esta idea descabellada es motivada por el rechazo a ceder a las sirenas de la razón o, en todo caso, de una cierta forma de racionalismo que nos invita a no ver sentido sino en la unidad. Ciertamente, la unidad puede tomar diferentes formas. Los filósofos han buscado "un principio, un sistema, una integración" o elementos, átomos, números imbricados para formar la unidad (Serres, 1982, p. 5). Pero según Michel Serres, nuestras tentativas para dar sentido al mundo al proyectar la unidad en la multiplicidad están destinadas al fracaso. En el fondo, ¿no estamos "tan poco seguros de lo uno como de lo múltiple" (Serres, 1982, p. 3)? Lo indivisible se revela composite; lo simple, complejo; lo irreductible recula ante nuestro análisis. Incapaces de captar lo elemental, nos volvemos hacia lo universal. Ahora bien, aún aquí la búsqueda parece desesperada. De decepción en decepción, hemos debido resignarnos a buscar las relaciones, considerando sus elementos individuales como intercambiadores y los sistemas como redes de conexiones. De acá en adelante, se considera que las relaciones tienen primacía sobre los seres, y que se trata de estudiar la estructura formal de los fenómenos más que su contenido (algo a lo que se prestan tanto la fenomenología como el estructuralismo). Michel Serres constata que la racionalidad que había fracasado en encontrar unidades ontológicas, simplemente ha sido transpuesta a esta búsqueda del sentido, y que ella arriesga una vez más con decepcionarnos. ¿Se puede hablar de un "átomo de relación"? (Serres, 1982, p. 4). Nada es más incierto. Incluso no estamos seguros de que se pueda hablar de relaciones en este sentido, como si

Cómo citar: Webb, D. (2019). Pensar lo múltiple sin el concepto: hacia un intelecto democrático. Ciencias Sociales y Educación, 8(16), 157-168. DOI: https://doi.org/10.22395/csye.v8n16a10

Traducción realizada por Luis Alfonso Paláu-Castaño. Agradecemos la editorial L'Herne permitir el acceso del libro en francés para su versión, en partes, en español.

Recibido: 10 de agosto de 2019.

Aprobado: 20 de septiembre de 2019.

** Profesor de filosofía en Staffordshire University, se interesa en la filosofía contemporánea europea. Autor de numerosas obras y artículos. Ha escrito especialmente sobre Michel Foucault, Miches Serres y Jean Cavaillès. Publicó Heidegger, Ethics and the Practice of Ontology (Continuum Press, 2009). 
toda relación en tanto que tal respondiese a una cierta determinación formal. En efecto, solo nos queda saber "cómo la relación se vuelve ser y el ser relación" (Serres, 1982, p. 4); no es suficiente con bascular de una ontología de la substancia a una ontología de las relaciones para resolver el misterio.

Como Bachelard y antes de él, Serres considera que la simplicidad y la unidad solo aparecen allí donde no se mira de bastante cerca. Son ilusiones debidas a una falta de atención al detalle de la experiencia, así como los conceptos pueden enmascarar ensamblajes más sueltos, "multiplicidades muy a menudo dispersas" (Serres, 1982, p. 3). Desde sus primeros escritos, Michel Serres ha luchado a brazo partido con este problema, su voluntad de mantener un anclaje en lo cotidiano hace de él el filósofo más resueltamente anti-platónico de los tiempos modernos. En desquite, para nada es polemista; siempre ha puesto cuidado en no definir el conocimiento ni como pleno ni vacío, total o nulo, pues "comúnmente sabemos un poco, más o menos, bastante, mucho" (Serres, 1982, p. 5). Nuestra existencia está atravesada por visiones y sonidos, por la historia, el tiempo, los colectivos, el barullo de la vida por nada puede ser reducida a una unidad. Sobre el fondo de esta toma de conciencia precoz, Génesis afirma la voluntad "de pensar lo múltiple tal cual, dejándole flotar sin detenerlo por la unidad, dejarlo libre" (Serres, 1982, p. 5). Es esta una empresa desconcertante que parece invocar tanto un concepto como una definición de la multiplicidad como unidad formal, y arriesga por ello mismo traicionar la intención del proyecto. Al exponer los medios para ponerlo a operar, Michel Serres adopta por lo demás una postura ambigua de la que no se podría decir si ella busca pensar lo múltiple directamente, o tomar distancia para reflexionar en las herramientas, en las ideas y los enfoques que lo permitirían. Una u otra aproximación serían profundamente problemáticas. Sin embargo, la distinción tradicional entre discurso y metadiscurso no le hace justicia a los propósitos de Michel Serres, que tiene más tendencia a diluir esta demarcación en una región compleja y dinámica. Lo que se puede leer como una metodología, como una teoría de la estructura subyacente a la propagación de lo múltiple, se revela indisociable de la realidad de la que ella da cuenta o informa. Veremos que esta aproximación, consecuencia del materialismo de Serres y más precisamente su atomismo, da lugar a lo que se podría llamar "ontología indirecta". Esta ontología indirecta se distingue por su aparente continuidad con la epistemología: las condiciones del ser no están radicalmente separadas de las condiciones del conocimiento (por razones completamente diferentes de las que valoriza el idealismo). En el fondo, pensar lo múltiple como se propone hacerlo Michel Serres, es lanzarse en una aventura que resiste radicalmente a la unidad y cuya ambición es abiertamente ética.

El pensamiento de lo múltiple encuentra ciertamente precedentes en la historia de la filosofía. Entre los más significativos conviene citar el atomismo, 
que ejerce una influencia importante sobre la obra de Michel Serres. Pero antes de llegar al atomismo, evoquemos brevemente el estudio que consagró a los modelos matemáticos de Leibniz y a la unidad que los sistematiza (Serres, 1968). Él remarca que un lector de Leibniz estará desorientado sin duda por la diversidad de enfoques propuestos: dinámico, lógico, ontológico, empírico, matemático... Tratándose de aprehender la totalidad, uno llega a preguntarse si esos enfoques son compatibles entre ellos, y cuál tiene la prioridad. Según Serres estas cuestiones no son pertinentes en Leibniz, para quien cada aproximación introduce las siguientes sin que ninguna sea fundamental o imponga a las otras un conjunto de limitaciones. Por ejemplo, en matemáticas la enseñanza de tal o cual disciplina puede conducir a presentar a esta como independiente, portadora de conceptos, de reglas y principios propios, indisolublemente ligados por la cadena del razonamiento deductivo. Ahora bien, en realidad este orden no es quizás más que una estratagema heurística que no da una imagen precisa de las matemáticas en tanto que tales, donde tal sub-disciplina e incluso aquel otro concepto, esa figura, tal número, son la encrucijada de aproximaciones diferentes y convergentes, "una constelación de vías de acceso, todas igualmente rigurosas, todas igualmente deductivas" (Serres, 1968, p. 12). Multiplicando así las cadenas de razonamiento posibles, los vínculos se encuentran reforzados más que debilitados, y el análisis se enriquece: el orden aparece como una red o un tejido de conexiones donde cada punto está conectado a los otros, en tanto delimita la totalidad en regiones distintas. Estas tienen valor de modelos para asir otras regiones, sin que por ello dicten las leyes que las rigen y que determinan uniformemente la totalidad. Por no poder demostrar una unidad formal se pone en duda la existencia de una totalidad o un sistema, pero la idea misma de totalidad continúa produciendo sentido en la medida en que cada uno de esos modelos sirve de clave para reunir la red más amplia de relaciones. La concepción matemática del sistema en tanto que totalidad no tiene que ver con una teoría matemática en particular ni la comprensión sistemática de los diferentes modelos (en tanto que unidad) equivale a modelizar el sistema siguiendo tal o cual teoría. Más bien: la concepción matemática del sistema como totalidad se sostiene en la relación sistemática de los modelos matemáticos. Solo Dios puede abarcar todas las variaciones posibles en una visión sinóptica de la totalidad; nosotros, seres finitos, debemos contentarnos con pasar de una a otra en una serie de iteraciones. Por mucho que esta finitud nos autorice aún a hablar de concepto, es "por una suerte de inducción, de paralelismo o de similitud" que "la localización iterada de diferentes modelos permite que aparezca la estructura que los une analógicamente" (Serres, 1968, p. 5). Concebir el sistema en su totalidad es establecer conexiones por medio del pensamiento, conectar diferentes regiones o modelos según diferentes puntos de partida y llegada. Este enfoque de la unidad le debe igualmente mucho a los trabajos matemáticos del grupo 
Bourbaki, en los que se ha inspirado la obra entera de Serres. Por ejemplo, veinte años más tarde en El Tercero instruido, Michel Serres (1991) escribe:

Cuando escucháis o componéis variaciones sobre un tema dado, ¿no os preguntáis a veces, si el tema mismo no se desarrolla como una variación entre otras? Más simple sin duda, más puro, más corto, cierto, pero ¿Por qué separarlo de ellas? Existe tanta distancia entre estas últimas como entre ellas y el tema que nada impide entonces que yo llame variación sobre una de las variaciones. ¿Por qué prejuzgarla más estable y mejor centrada que estas últimas? Sí, el tema no es más que una de las variaciones (p. 108).

Sin embargo, como lo había ya constatado en su lectura de Leibniz, Serres recuerda que no existe clave única para leer la serie que puede declinarse en muchos órdenes, siguiendo diferentes puntos de partida y diferentes itinerarios posibles hacia el ensamblaje de los elementos en un todo.

El concepto no es pues solamente un work in progress, es un periplo sin destinación precisa, con trayectorias forzosamente múltiples. En estas condiciones, ¿cómo reencontrarse allí? Orientarse "correctamente" en un sistema racional remite a la problemática más general del orden intrínseco de los procesos de transformación. Al sustituir la imagen de la línea por la de la red, como ya lo había hecho Leibniz, Michel Serres (1968) afirma que ningún proceso puede definirse en términos de secuencias lineales, por simples rupturas o cambios de valor: "Una transformación real es siempre esencialmente complicada" (p. 285). Con este espíritu, él sugiere escindir el proceso en muchos tiempos elementales y "proyectar la multiplicidad de esas líneas en un espacio de representación", donde ellas vendrán a circunscribir una superficie compleja que acoge esquemas puntuales de orden y relación. Puesto que el sistema en tanto que totalidad es el conjunto de las relaciones entre los modelos (y no una forma fija que determinaría esas relaciones), la correspondencia entre el sistema en tanto que totalidad y su formalización por el pensamiento se opera en un tiempo entendido como superficie compleja. El concepto — si es que hay necesidad todavía de emplear este término aquí- sería la forma en la que se presenta la multiplicidad, y no como en Kant una forma intemporal. En efecto, el concepto (que remite al sistema en tanto totalidad o a toda transformación) es por definición temporal y complicado. Como en un conjunto de variaciones, esta idea se declina a todo lo largo de la obra de Serres teñida de diversas influencias, pero siempre reconocible. La reencontramos en Génesis donde la apuesta consiste precisamente en pensar lo múltiple sin el concepto. Pero antes digamos algo sobre el atomismo.

En 1977, aparece El nacimiento de la física en el texto de Lucrecio. Caudales y turbulencias, bella meditación sobre el atomismo epicúreo expuesto en el De 
rerum natura (Serres, 1994). Michel Serres le atribuye allí a Lucrecio un materialismo radical y sorprendentemente moderno, según el cual las diversas manifestaciones del orden, ya sea el de las galaxias, el de los sistemas meteorológicos o del espíritu humano, en física, en historia o en moral, resultan todos de un solo y mismo proceso repetido a diferentes niveles, y que genera una serie potencialmente infinita de variaciones. El "modelo general" está compuesto de una infinidad de átomos que atraviesan el vacío siguiendo líneas paralelas. Sin causa y de forma totalmente imprevisible, la trayectoria de algunos átomos sufre ínfimas desviaciones (el clinamen), que provoca colisiones, turbulencias y torbellinos por los cuales los átomos y sus combinaciones vienen a adoptar un movimiento regular. Estos torbellinos forman el orden que observamos en el mundo, así como en todos los dominios de la vida natural, social, económica y moral. El orden es pues dinámico, es una recurrencia más o menos estable cuya disolución terminará por acelerarse. Entonces, llevados al flujo cósmico, los átomos resurgirán eventualmente en otra parte, en nuevos ordenamientos.

Puesto que el orden resulta de un proceso esencialmente aleatorio, ninguna ley explica cómo los átomos se combinan para formar grupos y estructuras estables. Las leyes de la física se presentan como el esquema de regularidad que emerge de ese proceso como una especie de alianza, forzosamente localizada en el espacio y el tiempo. Es muy probable que en nuestra región del mundo, las leyes de la naturaleza no sean las mismas que en otra parte, y puesto que el clinamen suscitará combinaciones nuevas e inesperadas, nuestras leyes estarán ellas mismas llevadas a cambiar. Esta localización en la emergencia del orden no vale por lo demás solamente para la realidad física. La teoría de Lucrecio lleva a la historia, al clima, a la moral, a la biología y a muchos otros dominios, al movimiento de los átomos en el vacío. Al estar desprovisto este movimiento de leyes fundamentales y universales (al estar el vacío privado de todas las propiedades métricas), no podríamos explicar esas superestructuras de orden reduciéndolas a una realidad más elemental de donde ellas emergerían. Igualmente, Michel Serres puede asimilar la moral a la física sin tener que ceder al reduccionismo físico. En otros términos, la localización que expresa la difusión de regularidades a nivel del mundo físico es reproducida a nivel de las realidades discursivas. Pasar de la física a la economía o de la historia a la moral, es pasar de una región del universo a otra. No hay rampa que permita pasar de lo local a lo global, ni camino balizado para pasar de una región local a otra, aunque toda región (física y discursiva) esté sometida a los mismos principios de los átomos movidos en el vacío, turbulencias, torbellinos.

El sistema forma una trama, pero puesto que no se parte de una totalidad que plantee las condiciones de posibilidad de cada parte, resulta que hay que anudar los lazos. 
El proceso material que hace emerger regiones locales distribuye semejanzas y diferencias. El isomorfismo entre regiones (físicas o discursivas) nunca es perfecto, y el movimiento analógico en el cual se tejen vínculos de uno con otro está destinado al inacabamiento. También el pensamiento y la escritura son actos materiales que tienen que ver con un proceso, que potencialmente llevan a una reconfiguración de las conexiones. En eco a la interpretación que Michel Serres propone del sistema leibniziano, se sigue que un sistema concebido como totalidad (o un sistema de regiones) es la relación entre todas las regiones. Ahora bien, para concebir el conjunto de las relaciones es preciso cartografiarlas. Porque no existe conjunto formal e invariante de relaciones que unifique las regiones en un todo, el resultado dependerá forzosamente del camino que se ha escogido trazar. La interpretación que Michel Serres propuso de Leibniz solo encuentra una débil resonancia en su interpretación de Lucrecio. Parecería que una desviación hubiera venido a torcer el camino que lleva del uno al otro. En Leibniz, reducir la totalidad a una concepción única aparecía como una tarea infinita, cuya posibilidad estaba garantizada por la existencia de Dios, y solo podía ser realizada por Él. En contrapartida, De rerum natura se propone elaborar una teoría del universo y de todo lo que contiene sin recurrir a un principio divino. No solamente Lucrecio se cuida bien de no ir a invocar un solo Dios único y todopoderoso, sino que su proceder mismo se lo prohíbe formalmente. En el universo lucreciano, un ser completo y perfecto que abarcara el concepto del universo como totalidad es imposible de pensar. Por consiguiente, cualquiera sea el ángulo de incidencia escogido para abordar el sistema, la forma que de ello emerge no es ni estable ni única. El universo, infinito y abierto, está constantemente expuesto a interacciones complejas con las fuerzas y las corrientes que lo flanquean. Una cartografía sigue siendo posible, pero ella será de acá en adelante resueltamente local. Para la teoría atomista, lo múltiple no reposa tanto en la noción de átomos (que considerados como ontológicamente independientes, seguirían siendo unidades), como sobre las posibilidades sin fin de variación, de formación y reformación a los que ellos se presten.

Pero regresemos a Génesis, donde los pocos jalones que acabamos de plantear nos ayudarán a comprender cómo Serres logra pensar lo múltiple sin el concepto, en particular en el capítulo decisivo intitulado "Nacimiento del tiempo".

A primera vista, el envite de este capítulo parece difícil de establecer. En resumen, Serres evoca en él la noción de lo múltiple, critica nuestra terquedad en perseguir diversas formas de unidad y propone una teoría del tiempo como emergencia de esquemas de repetición o iteración en un flujo caótico. Haciendo esto se expone a un doble riesgo: por una parte, se confronta directamente con la idea de multiplicidad arriesgando términos como pura multiplicidad (lo que parecería traicionar su intención de no recurrir al concepto); por otra parte, 
aborda una inmensa variedad de temas, recurre a una terminología fluctuante dando testimonio de cierta reticencia a conducir el análisis sistemático que el lector hubiera podido esperar de una teoría de la multiplicidad en tanto que tal $^{1}$. Sin embargo, ninguna de estas dos críticas es pertinente; en pensamiento como en palabra, el compromiso de Serres con respecto a la multiplicidad es particularmente fuerte, sobre todo con miras a la reciente preocupación de la filosofía por el tema.

En medio de ese capítulo, Serres se apoya en la experiencia cotidiana para establecer que el tiempo es a la vez continuo y discontinuo, se despliega y se contrae, pasa y no pasa; en una palabra, parece desafiar toda formalización. Lagunar y esporádico, mosaico esparcido, el tiempo es una especie de multiplicidad que comprende poco o nada unidades discretas, pero presenta un cierto grado mínimo de repetición o redundancia. En otros términos, la posibilidad de la repetición no está aquí del todo ausente. No es un flujo que se diferencia en fluxiones, aunque pueda devenirlo; no es un conjunto, aunque pueda llegar a serlo. Si la redundancia mínima aumenta y la multiplicidad deviene más ordenada, el tiempo se cristaliza en un espacio o en espacios. Por ejemplo, el tiempo es el umbral entre desorden y redundancia del espacio. Es "la primera inyección de redundancia en una pura multiplicidad" (Serres, 1982, p. 79).

Por pura multiplicidad Serres designa el puro caos, la multiplicidad caótica o la noise. Más allá de los límites de la indeterminación, la noise es un análogo del infinito, un fondo donde el orden temporal emerge bajo la forma relativamente finita de una "multiplicidad ordenada", sin equivaler a la negación, como si manifestase una lógica interna o estuviese separada de lo no-temporal por un límite claro. La concepción de la multiplicidad está suspendida entre dos extremos: multiplicidad caótica que resiste a toda determinación y multiplicidad ordenada de una colección de elementos bien definidos. Ella ocupa una región intermedia que es esencialmente temporal hasta que su variación se fije poco a poco en las proporciones y esquemas fijos que caracterizan el espacio. Pero como esta identificación no es definitiva, el tiempo no desaparece nunca completamente, entonces concebir el orden o la identidad implica también aprehender la noise como principio irreductible del desorden creador.

Para Michel Serres pensar lo múltiple es comprometerse en esta región intermediaria del tiempo sin determinar frontalmente su estructura. Lo múltiple

\footnotetext{
En el mismo capítulo Serres (1992) evoca la empresa de clasificación (cita a Linneo, Dumézil y Marx), la noción de furor (Júpiter, Marte, Quirinus), la historia (Roma, el objeto, la sociedad, la India), el proceso (Darwin, el tiempo), el caos, las sacudidas, el motín (la figura del filósofo, las fluxiones, de nuevo la historia, las turbulencias), el trabajo de lo múltiple (muchedumbres, olas, nubes, meteorología, ciencia, filosofía), las intermitencias (Platón, las galaxias), los sólidos y los fluidos (Lucrecio, Bergson, otra vez el tiempo y las turbulencias), los mixtos (lo uno y lo múltiple, Leibniz, el humo, el universo, la multiplicidad pura y ordenada, las faces), el tiempo (caos, turbulencia, expansión y contracción, discontinuidad-continuidad, envejecimiento, noise, turbulencia).
} 
es aprehendido como una mezcla de multiplicidad pura y ordenada que al no tener forma general, será descrita en su especificidad. Estas descripciones por lo demás no tienen nada de fijas en la medida que Serres se interesa ante todo en su carácter dinámico, en su modo de desarrollo y acción. Una mezcla se sitúa por consiguiente a medio camino entre la estructura estable dotada de una forma identificable, y el caso particular siempre sometido a variaciones. Cada nuevo detalle sacado a luz en una mezcla, es susceptible de cambiar desde que sea encarado con respecto a otras mezclas o examinado de cerca ${ }^{2}$. A diferencia del conocimiento ante-predicativo desarrollado por la fenomenología, este proceso está abierto y sin fin. De este modo el capítulo "Nacimiento del tiempo" invita a repensar las clasificaciones rígidas de la religión, la sociología y la antropología bajo la modalidad de etapas temporales de la evolución darwinista. Si se mira de cerca, se pueden reencontrar los esquemas de la evolución darwinista operando en la religión, la sociología y la antropología, y su formalización a partir de las teorías biológicas de Darwin no son sino una de las rutas posibles para explorar la relación entre esas diferentes disciplinas. Evocando los principios de soberanía, combate y producción encarnados respectivamente por Júpiter, Marte y Quirinus, Michel Serres remite al análisis de Dumézil según el cual el "furor" (o lo que Serres llama la noise) aparece como su rasgo común, el motivo que persiste en ellos y donde nacen. En el caso de la historia política, el conflicto no encuentra sus orígenes en las clases, sino que son las clases las que vienen a apaciguar un furor preexistente ${ }^{3}$. La noción misma de clase es un "efecto de umbral", se constituye con respecto al ruido, al furor a los que ella da provisionalmente una expresión ordenada. A título de ejemplos, Serres cita igualmente la construcción de barreras, el lenguaje científico y la administración, donde las clases codifican un proceso que Serres llama violencia, río, vida o invención. Descripciones filosóficas y científicas de una codificación pueden seguir siendo posibles, pero solo fijan el proceso en el dialecto de la clasificación que caracteriza regiones específicas, es decir, en un idioma que aspira a la pureza en detrimento de sus relaciones con las fuerzas discursivas que lo rodean. Es para contrarrestar esta tendencia que el autor elige interesarse en las mezclas. Resiste a la tentación de la unidad y el ideal para trazar la filiación del pensamiento con la noise de la que él emerge, donde el entendimiento flanquea la sensibilidad, el tiempo marca la juntura entre la multiplicidad caótica y el ordenamiento espacial de los conceptos y categorías. Sin duda, es para cultivar esta proximidad que él está resuelto a prescindir del concepto en el sentido tradicional.

\footnotetext{
2 Se reencuentra acá el motivo del "tercero", ese lugar excluido por una lógica dominante en un lugar y en sitio dados. De él se trata ampliamente en el Tercero-instruido, pero aparece por primera vez en un capítulo del Hermes I: la comunicación (Serres, 1969).

3 Serres (1982) compara explícitamente el furor con la guerra hobbesiana de todos contra todos. Sin embargo, la "guerra" es ordenada, preparada, institucionalizada (p. 57).
} 
Si el rechazo del concepto como forma unificadora remite a Leibniz (para quien el sistema emerge a través de la iteración de los modelos), remite igualmente a Lucrecio (que rechaza toda idea de sistema como totalidad, considerando que la multiplicidad se propaga a partir de los esquemas puntuales de orden hasta procesos desordenados de donde ellos emergen). Pero si con referencia a Kant, se le asigna a Michel Serres la intención de recoger intuiciones sin conceptos, será necesario reconocer la absurdidad de esta empresa desde el momento en que la síntesis de las intuiciones y los conceptos es atribuida a la conciencia trascendental. Contra esta suposición, la influencia del atomismo lucreciano persuade a Serres de que el trabajo de la síntesis es un proceso material. El "proceso puro" que busca pensar es el tiempo de los mundos y las cosas, el tiempo de la vida, enzarzado en combinaciones que se aflojan progresivamente (Serres, 1982, pp. 65-66). Pensar este proceso en tanto tal, no implica que se renuncie a todo concepto y toda forma de unidad, como si el entendimiento pudiera liberarse de sus propios comienzos; es hundir el pensamiento en las aguas turbulentas donde se forman los conceptos y las unidades. Es situar el pensamiento lo más cerca de la pura multiplicidad, de la noise, sin nunca imponerle una estructura ni demolernos con ella en el caos. Se podrá objetar que todo esto es muy bello, pero el uso que Serres hace de términos como caos, noise y turbulencia le ofrece una especie de metalenguaje que lo lleva a pesar de todo a describir la multiplicidad. Sin embargo, esta objeción no es pertinente en tanto que la significación de estos términos en la obra de Serres emerge de un medio particular. Cuando es cuestión de turbulencia a propósito del clima, del lenguaje, de la historia o del pensamiento, Serres echa mano de una idea formada en un cierto contexto y se sirve de ella para trazar la codificación de otra. La turbulencia que desprende el análisis de los sistemas meteorológicos no es la turbulencia de la historia, del lenguaje o el cuerpo. De la misma manera, la "multiplicidad" no constituye un solo y mismo concepto bajo el cual se filarían todas las ocurrencias de lo múltiple. A pesar de su fecundidad, la teoría formulada por Riemann, y retomada por Husserl y Bergson en la tradición filosófica, no es sino una teoría entre otras, por completo independiente de esta concepción matemática, donde se podría encontrar un sentido de la multiplicidad en la muchedumbre, en el lenguaje, en la tormenta y en muchos otros fenómenos. Para Serres, esas ocurrencias no son reductibles las unas a las otras, y ningún sentido tiene prioridad sobre los otros. Plantear una precedencia equivaldría a exigir que las cosas en tanto que existen, se conformen con la misma condición de unidad e imponer una sola forma de relación entre los elementos de todas las multiplicidades. Esto conduciría a aplicar una ontología formal de la diferencia. Serres evita este escollo y muestra por el contrario que la relación, y por tanto la diferencia entre los elementos de los diversos múltiples es ella misma variable. Por consiguiente, cuando Serres evoca la multiplicidad a través de las masas, 
las nubes, los movimientos históricos, las clases (otras tantas formas de mezclas temporales), no describe simplemente un agregado particular, hace signo hacia el tipo de diferencia que este encarne y difunde.

Se podría creer que este entorno fluctuante ofrece un campo de juego en el cual no importa qué determinación conceptual terminará por dar en el clavo. A pesar de ello, la escritura de Serres prueba ser rigurosa. Y ello tiene que ver especialmente con la atención que le pone a los matices que separan las regiones del pensamiento y la escritura. A medida que los términos se desplazan de un contexto a otro, transportan algo de su proveniencia material a las nuevas relaciones que forman, donde la transferencia terminológica testimonia una transferencia estructural más amplia, ella misma en mutación constante. El materialismo de Serres es tal que los términos vectores son sacados de lo más próximo, en ejemplos concretos sacados de lo cotidiano (literatura, arte y ciencias). Si fuera de otra manera, delataría una aspiración hacia el idealismo que sería contrario al espíritu mismo de su obra. Por otra parte, este materialismo conduce una deformación y reforma del discurso por el movimiento de los términos transferidos, por tanto una mutación constante del carácter formal de la experiencia. Michel Serres no pretende llegar a una determinación final de los grupos, los agregados, las multitudes y los flujos, puesto que no puede definirlos como totalidades o unidades sin descuidar el desorden y variación que continúan atravesándolos, sin desatender en el mismo movimiento la porosidad compleja de sus fronteras. Responder a esta circulación del desorden, a esta turbulencia, es (en los propios términos de Serres) "negociar el ruido" del que todo orden está impregnado (Serres, 1982, p.91), y el grado en el que alguien o algo (una ciencia o una tecnología) sea capaz de hacerlo da la medida de su inventividad. La incapacidad para inventar nos condena a la redundancia de un proceso rígido, de un esquema inflexible, de una historia calcificada, al refugio del instinto o las instituciones. Por el contrario, la creatividad exige que uno se coloque en el proceso material de síntesis, lo más cerca posible de la noise y el caos, en un punto donde las cosas y el lenguaje forman esquemas de redundancia. Esto puede incitar a formar nuevos esquemas de relación, pero también conduce a desorganizar a los que se han esclerosado y obstaculizan la comunicación. Michel Serres (1982) considera que el filósofo es el mejor colocado para entregarse a esta práctica que consiste en regular la libertad que acompaña la ruptura del orden, tanto como para liberarse de las reglas (p. 71). En este sentido, "regular la libertad" no consiste en legislar —en el sentido ortodoxo de aplicar una ley para regir un conjunto de fenómenos posibles (y definirlos como posibles)—, sino más bien intervenir en una situación de desorden relativo de manera que se promueva la formación de regularidades, mientras traza relaciones analógicas entre dominios, aclara la operación de los conceptos en diferentes regiones discursivas o esboza esquemas de regularidad por el 
pensamiento y la escritura. Se trata de sumergirse en esas regiones donde el orden que comienza a emerger o está a punto de derrumbarse, presenta signos de movimiento a los que el pensamiento puede darles un nuevo impulso. Así concebida, la inventividad no se reduce a la simple modificación, no tiene nada que ver con una creación ex nihilo "de la nada"; procede del reconocimiento que las cosas están generalmente mal adaptadas a las identidades que tenemos el hábito de atribuirles, y ellas son a la vez algo más o menos de lo que permiten las condiciones formales de su existencia. Conviene comenzar por las mezclas, los híbridos impuros a la vez animados y desestabilizados por la noise que los habita y rodea. Es necesario aceptar dejarlos hablar en una multitud de lenguas, codearse con otros idiomas. Así, el pensamiento de lo múltiple supone un compromiso inventivo con respecto a la noise, que nos lleve a cartografiar regiones hasta entonces inexploradas entre identidades conocidas (el tercero) y ponerlas en relación, la menos violenta o la menos caótica con el orden circundante.

Esta empresa demanda una combinación inhabitual de perspicacia filosófica e ingenuidad estudiada. Se trata de tomar las cosas como vienen, sin agarrarse al concepto, al lenguaje y la regla como a verdades inmutables en un océano de confusión (Serres, 1991, p. 46). Hemos visto que esto se aplicaba igualmente bien a términos como multiplicidad, noise y turbulencia, recurrentes en la obra de Serres. Las palabras son cosas y su significación es también intrínsecamente variable. La reticencia de Serres a abandonar el terreno de la experiencia es una reticencia a abandonar el lenguaje, y refleja la intuición que las cosas no se presentan en el lenguaje, como si se pudiera esperar remontar a su origen (para Serres esto es una inepcia (1991, p. 56)). En la teoría atomista, el orden mismo es un código: "las cosas se presentan como portadores de su lengua" (Serres, 1994, p. 147). Escribir sobre las cosas es participar en su metamorfosis, en el curso de un viaje a través del lenguaje y entre más grande sea nuestro compromiso con ellas, más rica será nuestra experiencia. "Para crear es necesario saberlo todo y por tanto haber trabajado inmensamente" (Serres, 1991, p. 72), escribe Serres antes de añadir que eso no es suficiente para alimentar la inventividad. Para resistir a la fuerza de clasificación, nos lanza esta orden expresa: "apréndelo todo primero, después, llegado el día, echa al fuego todo lo que posees incluidos tus zapatos, ve en ese simple aparato" (Serres, 1991, p. 74). Las estructuras del pensamiento crítico, en tanto que configuraciones materiales, están ellas mismas aferradas a relaciones fluidas con estructuras que regulan más visiblemente nuestra libertad. Por consiguiente, pensar la multiplicidad sin el concepto, no es pensar libremente en los límites de la clasificación, sino vivir libremente rehusando abrigarse tras las instituciones, los media y las modas (Serres, 1991, p.69). Es un esfuerzo apasionado que compromete al cuerpo tanto como al espíritu y que orienta nuestra relación con el otro. El desafío epistemológico planteado por la ontología atomista, requiere una escena que combine la disciplina y la 
irreverencia, la atención meticulosa por el detalle y la vivacidad de espíritu, la pasión y la distancia con respecto al instinto. Esta ética nos llama a vivir en el espesor apretado de las cosas lejos de las cumbres. Serres (1982) la define como una tentativa a decir: "la simple felicidad de vivir en común en el seno de tal ciudad, el sutil placer de inventar en la pluralidad su conducta, su lengua, su obra singular y su existencia privada, su propio cuerpo" (p. 92). Estas invenciones no son de ninguna manera introspectivas, pues la escritura es el producto de un ruido de fondo, del cuerpo, de nuestros amores y nuestro compromiso con respecto al otro. Volver a trazar y consolidar las relaciones que establecen órdenes cuasi estables en el seno de un sistema abierto, es responder a la complejidad de las circunstancias locales y abrir nuevos dominios de los que podamos hablar, escribir y actuar. La ontología indirecta de Michel Serres (1991) como esfuerzo por pensar lo múltiple sin concepto, contribuye al desarrollo de lo que él llama "un intelecto simplemente democrático" (p. 69).

\section{Referencias}

Serres, M. (1968). Le Système de Leibniz et ses modèles mathématiques. París: Presses Universitaires de France.

Serres, M. (1969). Hermes I: la comunicación. París: Minuit. (Obra original publicada en 1963).

Serres, M. (1982). Genese. París: Grasset.

Serres, M. (1991). Le Tiers-instruit. París: F. Bourin.

Serres, M. (1994). El Nacimiento de la física en el texto de Lucrecio. Caudales y turbulencias. Valencia: Pre-textos. 